University of Rhode Island

DigitalCommons@URI

Mechanical, Industrial \& Systems Engineering

Faculty Publications

2020

\title{
Experimental Control and Statistical Analysis of Thermal Conductivity in ZnO-Benzene Multilayer Thin Films
}

\author{
Fabian Krahl \\ Ashutosh Giri \\ University of Rhode Island, ashgiri@uri.edu \\ Md Shafkat Bin Hoque \\ Linda Sederholm \\ Patrick E. Hopkins
}

See next page for additional authors

Follow this and additional works at: https://digitalcommons.uri.edu/mcise_facpubs

Creative Commons License

(c) (i)

This work is licensed under a Creative Commons Attribution 4.0 License.

\section{Citation/Publisher Attribution}

Krahl, F. et al. (2020) Experimental Control and Statistical Analysis of Thermal Conductivity in ZnO-Benzene Multilayer Thin Films. The Journal of Physical Chemistry. https://dx.doi.org/10.1021/ acs.jpcc.0c06461

This Article is brought to you for free and open access by the Mechanical, Industrial \& Systems Engineering at DigitalCommons@URI. It has been accepted for inclusion in Mechanical, Industrial \& Systems Engineering Faculty Publications by an authorized administrator of DigitalCommons@URI. For more information, please contact digitalcommons-group@uri.edu. 


\section{Authors}

Fabian Krahl, Ashutosh Giri, Md Shafkat Bin Hoque, Linda Sederholm, Patrick E. Hopkins, and Maarit Karppinen 


\title{
Experimental Control and Statistical Analysis of Thermal Conductivity in ZnO-Benzene Multilayer Thin Films
}

\author{
Fabian Krahl, Ashutosh Giri, Md Shafkat Bin Hoque, Linda Sederholm, Patrick E. Hopkins, \\ and Maarit Karppinen*
}

Cite This: https://dx.doi.org/10.1021/acs.jpcc.0c06461

Read Online

ACCESS |

山ll Metrics \& More

回 Article Recommendations

Supporting Information

ABSTRACT: We have fabricated a model system of precisely layer-engineered inorganicorganic thin-film structures using atomic/molecular-layer deposition (ALD/MLD). The samples consist of nanoscale polycrystalline $\mathrm{ZnO}$ layers and intervening benzene layers, covering a broad range of layer sequences. The samples characterized in this study combined with previous publications provide an excellent sample set to examine thermal transport properties in inorganic-organic thin films. The cross-plane thermal conductivity is found to depend on multiple factors, with the inorganic-organic interface density being the dominating factor. Our work highlights the remarkable capability of interface engineering in suppressing the thermal conductivity of hybrid inorganic-organic materials, e.g., for thermoelectric applications.

\section{INTRODUCTION}

Thermal conductivity is one of the critical properties for thermoelectric (TE) materials. An efficient TE material should have as low thermal conductivity as possible but simultaneously conduct electricity very well; this conundrum in optimizing TE materials is described by the so-called "electron crystal-phonon glass" concept. ${ }^{1}$ Advances in nanostructuring have rekindled the hope that materials could be engineered such that thermal conductivity could be reduced without simultaneously degrading the electrical conductivity. ${ }^{1,2}$ Here, we focus on heat conduction and investigate the possibility to manipulate thermal conductivity by designing nanoscale multilayer thin-film structures.

Heat is conducted in solids by both charge carriers and phonons, which are quantized lattice vibrations. In dielectric materials, the influence of charge carriers on the heat conduction is negligible, and the overall thermal conductivity is mainly driven by the lattice thermal conductivity, $\kappa_{\mathrm{L}}$, described by the kinetic theory as

$$
\kappa_{\mathrm{L}}=\frac{1}{3} C_{\mathrm{V}} \bar{v} l_{\mathrm{mfp}}
$$

where $C_{\mathrm{V}}$ is the specific heat capacity, $\bar{v}$ is the group velocity of the phonons, and $l_{\mathrm{mfp}}$ is the mean free path a phonon takes before being scattered. ${ }^{3}$ This particle-like scattering at material interfaces together with the wave nature of phonons, showing coherence of heat-carrying vibrations across multiple interfaces in short-period superlattices (SLs), has led to the realization of tunability in the thermal conductivity in a wide range in these superlattices through efficient engineering of the mean free path of the phonons. ${ }^{4-8}$ Earlier works on crystalline inorganic-inorganic SL systems have shown remarkable reductions in heat conduction, demonstrating ultralow thermal conductivity values even surpassing the alloy limit to thermal conductivity of the constituent materials of the SL structures. $^{5,7-24}$ This reduction in thermal conductivity is not limited to purely crystalline interfaces, as crystalline-amorphous SLs have also revealed promising results demonstrating ultralow thermal conductivities well below the alloy limit. ${ }^{25-27}$ This widens the variety of interesting model systems that can potentially possess the ability to tune their thermal properties over a wide range and opens a plethora of opportunities to study the underlining mechanisms controlling heat conduction in novel superlattice-type structures.

Compared to inorganic-inorganic systems, hybrid inorganic-organic SLs are intriguing in the sense that they combine components that are fundamentally different. Indeed, for such inorganic-organic interfaces, large acoustic impedances between the inorganic and organic materials have been observed, ${ }^{28-30}$ resulting in a considerable reduction in the thermal boundary conductance across the inorganic-organic interface. Organic molecules have been successfully intercalated, for example, into $\mathrm{TiS}_{2}$ and related two-dimensional (2D) lattices from organic solutions both electrochemically and chemically to suppress their thermal conductivity. ${ }^{31-34}$ However, this approach does not allow the control of the number/frequency of the organic layers within the inorganic matrix.

Received: July 15, 2020

Revised: October 13, 2020 
The gas-phase atomic/molecular-layer deposition (ALD/ MLD) technique ${ }^{35,36}$ is uniquely suited for the fabrication of precisely layer-engineered inorganic-organic thin-film structures. This technique is derived from the state-of-the-art atomic layer deposition (ALD) thin-film technology of highquality ultrathin inorganic thin films. ${ }^{37}$ It allows us to combine, under relatively mild deposition conditions, mutually reactive inorganic and organic precursors into hybrid materials. Furthermore, ALD/MLD provides an atomic/molecular-level control of the individual-layer thicknesses for any predesigned layer sequence pattern.

Here, we investigate the cross-plane thermal conductivity of $\mathrm{ALD} / \mathrm{MLD}$-fabricated $\mathrm{ZnO}$-benzene thin films in the thickness range of 50-200 nm, composed of polycrystalline $\mathrm{ZnO}$ layers and monomolecular benzene layers or few-layer $\mathrm{Zn}-$ benzene pilings. This material system is chosen for several reasons. The $\mathrm{n}$-type semiconducting $\mathrm{ZnO}$ is a reasonably good thermoelectric material as such, especially when doped with, e.g., $\mathrm{Al}$ or $\mathrm{Mg}^{38-42}$ and its deposition from diethylzinc (DEZ) and water is one of the prototype ALD processes. ${ }^{43}$ Most importantly, the thermal conductivity in $\mathrm{ZnO}$ is dominated by phonons (instead of charge carriers), ${ }^{44}$ which makes the introduction of organic interfaces a particularly effective approach for the thermal conductivity reduction in $\mathrm{ZnO}^{28,45,46}$ Ultimately, by adding increasingly more interfaces, we would approach $\mathrm{Zn}$-benzene films without dedicated $\mathrm{ZnO}$ layers; for such amorphous ALD/MLD-grown films, low thermal conductivity ${ }^{47}$ and high electrical conductivity ${ }^{48}$ have been reported. Along with the exceptional tunability in the thermal conductivity, the ALD/MLD-fabricated $\mathrm{ZnO}$-benzene thin films are mechanically flexible and easy to apply as a conformal coating even on textile fibers, which positions them as promising candidates for wearable energy-harvesting applications. $^{49-51}$

We have demonstrated the feasibility of the concept in our previous works, showing that the thermal conductivity of $\mathrm{ZnO}$ thin films can be reduced by a factor of 50 by inserting benzene layers within the $\mathrm{ZnO}$ matrix $^{28,45,46}$ and revealing that the insertion pattern needs not necessarily be regular to achieve the maximal reduction. ${ }^{46}$ Similarly, significant reduction in thermal conductivity was also seen for ALD/MLD-grown $\mathrm{TiO}_{2}$-benzene SL thin films. ${ }^{28,52,53}$ Recent reports suggest that different organic interfaces in $\mathrm{ZnO}$ may have different effects on the grain orientation, ${ }^{54}$ so the present work limits itself to $\mathrm{ZnO}-$ benzene interfaces.

We systematically evaluate the effect of structural/dimensional factors such as interface density, thickness, and structure on the thermal conductivity by fabricating and characterizing a series of multilayered samples with different film thicknesses and benzene-insertion patterns. In particular, the fact that the present extensive sample series is fabricated and characterized in essentially the same way as our previous $\mathrm{ZnO}$-benzene multilayer films allows for a reliable comparison between the new and previously published data, and the use of statistical data analysis techniques to address the importance of the different structural/dimensional factors in determining the thermal conductivity.

\section{METHODS}

2.1. Thin-Film Depositions. For thin-film growth, we used a hot-flow ALD reactor (Picosun R-100); this is the same setup also used in our previous paper. ${ }^{46}$ The precursors were diethylzinc (DEZ; >52 wt \% Zn basis from Sigma-Aldrich) and deionized water for the ALD process, forming $\mathrm{ZnO}$ layers, and hydroquinone (HQ; $\geq 99.5 \%$ ReagentPlus from SigmaAldrich) as the MLD precursor for the benzene layers. The purging and transport gas was nitrogen produced from an inhouse nitrogen generator (Parker HPN2-5000C-L-230V). The water and DEZ precursors were in bottles that were attached to the reactor at room temperature, while HQ was placed in a small heated glass cylinder that was connected to the reactor. Because HQ is a powder and even at the low pressures within the reactor (ca. $10 \mathrm{hPa}$ ) does not have a notable partial pressure, it needs to be heated up to $150{ }^{\circ} \mathrm{C}$ and kept at that temperature during the deposition. We deposited films simultaneously on a Si substrate $\left(3 \times 3 \mathrm{~cm}^{2}\right.$, prepared from Okmetic p-type Si wafers cut parallel to the (100) plane), a sapphire substrate $\left(1 \times 1 \mathrm{~cm}^{2}\right.$, MTI, cut parallel to the $(001)$ plane $)$, and a borosilicate glass $\left(3 \times 9 \mathrm{~mm}^{2}\right.$, prepared from "borofloat" from Finnish special glass) substrate. The precursor pulse/purge times were $0.3 / 5 \mathrm{~s}$ for the DEZ pulse, $0.5 / 5 \mathrm{~s}$ for the water pulse, and $15 / 30 \mathrm{~s}$ for the HQ pulse. The exact cycle sequences for all samples can be found in Supporting Information Table S1.

2.2. Thickness and Crystallinity Measurements. The film thickness was measured on the $\mathrm{Si}$ substrates with X-ray reflectivity (XRR) (Panalytical X'Pert Pro, $\mathrm{Cu} \mathrm{K} \alpha$ radiation generated with $40 \mathrm{kV}$ and $45 \mathrm{~mA}$ ). The thick films were crosschecked with spectral ellipsometry (J.A. Woollam M-200UI with an XLS-100 light source). This cross-checking is important for the films that are not regular superlattices because all nonsuperlattice films show very irregular and overlapping Kiessig fringes, making the thickness determination challenging with XRR alone. For the $200 \mathrm{~nm}$ films, the individual fringes are much smaller than for the $100 \mathrm{~nm}$ samples and were often unsuitable to determine the thickness in our XRR due to the limited resolution, but the SL peaks could be utilized to measure the thickness instead. The same diffractometer was also used for grazing incidence X-ray diffraction (GIXRD) measurements to confirm that the films are crystalline.

2.3. Thermal Conductivity Measurements. The crossplane thermal conductivities were measured using the timedomain thermoreflectance (TDTR) technique (see refs 55-57 for further details on the method). In our TDTR setup, a Ti:sapphire pulsed laser with a central wavelength of $\sim 800 \mathrm{~nm}$ is split into a $400 \mathrm{~nm}$ high-energy pump laser and an $800 \mathrm{~nm}$ low-energy probe laser. An electro-optical modulator (EOM) modulates the pump laser at $8.8 \mathrm{MHz}$ to create periodic temperature oscillations at the sample surface, and the resultant reflectance change is detected by a photodetector and a lock-in amplifier. By monitoring the ratio of the in-phase and out-of-phase signals $\left(V_{\text {in }} / V_{\text {out }}\right)$ from 0.1 to $5.5 \mathrm{~ns}$, the thermal properties of the samples were measured. All samples consisted of three layers: an Al transducer (nominal thickness, $80 \mathrm{~nm}$ ), the sample, and the sapphire substrate. The measurements were done using a $10 \times$ objective with coaxially focused pump and probe laser $1 / \mathrm{e}^{2}$ diameters of $\sim 18$ and 10 $\mu \mathrm{m}$, respectively. For analyzing the thermal conductivity, we followed the same procedure described in our previous publications. ${ }^{28,46,58}$ The uncertainty associated with the results originate from the transducer and sample thicknesses, $\mathrm{Al}$ thermal conductivity, sample heat capacity, and the $\mathrm{Al} /$ sample interface conductance.

2.4. Multivariate Data Analysis. Statistical data analysis to address the correlations between the structural/dimensional 


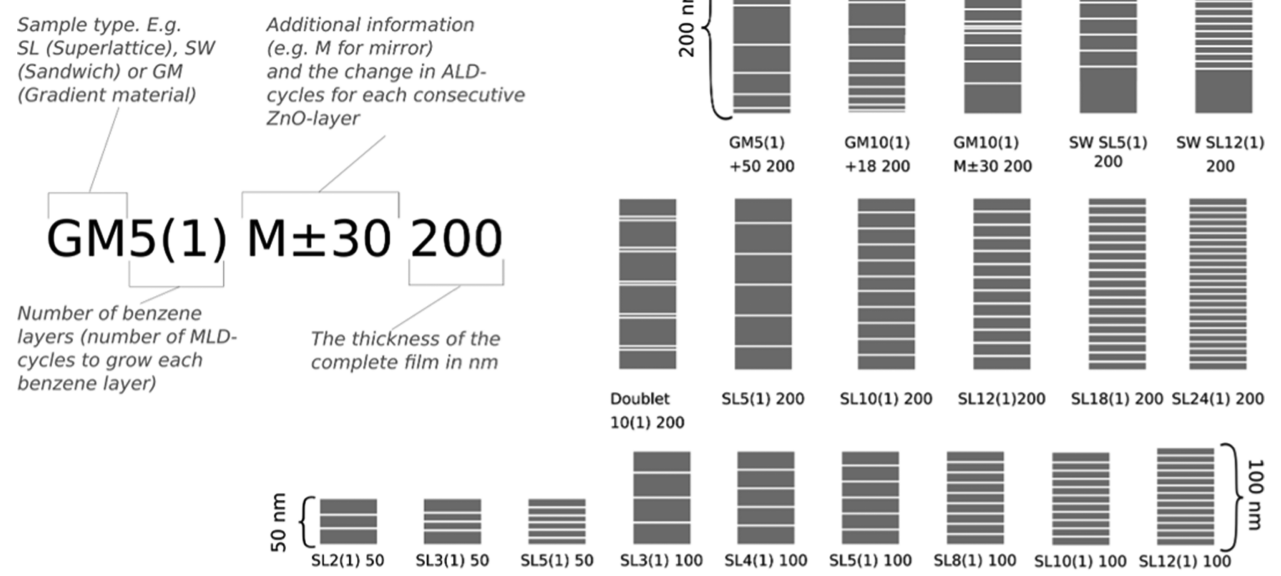

Figure 1. Cross-sectional internal structures of the newly deposited films (not to scale).

parameters ( $X$-variables) and the thermal conductivity ( $Y$ variable) was performed using the licensed multivariate analysis software Simca v.16 (Sartorius Stedim Biotech, Sweden, 2019). The partial least square ((PLS) projections to latent structure) regression method (included in the software) was selected for its ability to correlate multiple $X$ variables (in our case 8) to one or multiple $Y$-variables (in our case 1) in a manner analogous to a multidimensional trendline fitting. ${ }^{59-61}$ The original dataset consisting of 39 samples, each described by eight $X$-variables and one $Y$-variable, can be imagined as a nine-dimensional space where the samples are located based on the values of their $X$ - and $Y$-variables. In the PLS regression analysis, the data are projected to new axes (in our case 2) that are linear combinations of the initial variables, and orthogonal to each other. To visualize the data, the samples are projected to the two-dimensional plane formed by these two axes. Most importantly, in this regression process, the original variables receive different coefficients according to their contribution to the model.

In the PLS regression, the new axes of the projection are formed following the principles that (a) each new axis is added to represent the strongest trend among the observations that is not yet described by the other axes, (b) all axes are orthogonal, and (c) the best possible goodness-of-fit parameters are obtained. Following these principles, the model's axes are unambiguously defined in order of largest variance captured among the $X$-variables and strongest correlation with the $Y$ variables. The goodness-of-fit parameters describe the concordance and accuracy of the model: R2X and R2Y express how well the variance of the samples has been described, and Q2 expresses how well the model correlates the $X$-variables to the $Y$-variables (model linearity). These parameters thus set a limit to how many axes are useful to include in the model; in the present case, two axes were enough. A good model expresses goodness-of-fit parameters of at least 0.5 , while values of 0.9 and higher signify excellent model quality. Finally, successful model creation requires that the dimensionality of the statistical system is significantly reduced, preferably by at least half and to no more than four axes. $^{62}$

\section{RESULTS AND DISCUSSION}

In our analysis, altogether 39 samples were considered; among these, 20 samples were specifically synthesized for the present study (sketched in Figure 1), while 19 samples were reported in our prior works. ${ }^{28,45,46}$ These $\mathrm{ZnO}$-benzene structures differ from the commonly investigated epitaxial inorganicinorganic SL thin films in some important key aspects: (i) the interface is between an inorganic crystal and an organic molecule, (ii) the inorganic $\mathrm{ZnO}$ layers are polycrystalline, (iii) the organic layers are mostly one benzene-molecule thick, and (iv) they do not necessarily provide complete separation for subsequent $\mathrm{ZnO}$ layers. ${ }^{63}$ Additionally, we fabricated so-called gradient-material (GM) samples with a gradient spacing between the interfaces instead of the regular distance in superlattices to identify coherent phonon transport effects. ${ }^{5,7,8}$

The samples are systematically named according to the scheme presented in Figure 1. Our set includes regular SL thin films, GM thin films in which the spacing between the benzene layers is not constant, and also some special cases where, for example, superlattices are sandwiched (SW) between two thicker layers of $\mathrm{ZnO}$. The overall thickness of most films is around $100 \mathrm{~nm}$, but we also deposited thinner $(50 \mathrm{~nm})$ and thicker $(200 \mathrm{~nm})$ films along with $200 \mathrm{~nm}$ SW samples, which serve to compare and check the influence of interface density versus the total number of interfaces to the overall thermal conductivity of the films. Figure 1 shows the cross section of all samples fabricated for this study; a comprehensive list of all samples that includes thickness characterization results can be found in the Supporting Information (Table S2).

In Figure 2, XRR patterns are displayed for representative samples. The patterns show clear signals indicating good specular reflection from the surface and strongly suggesting smooth films with low surface roughness. In most superlattice samples, the SL peaks (indicated by small arrows in Figure 2) can be clearly seen, confirming that we indeed have sharp interfaces in regular distances. Contrary to our SL films that are easily identified by these SL peaks, the GM samples show no such regular pattern (see GM5(1)+50 200 in Figures 1 and 2). This confirms that their internal structure has no repetition and therefore deviates strongly from the superlattices. Clearly seen is also how the fringes become smaller with increasing film thickness (compare, e.g., the $50 \mathrm{~nm}$ samples with the 100 nm samples in Figure 2) and how the SL peaks still show up 


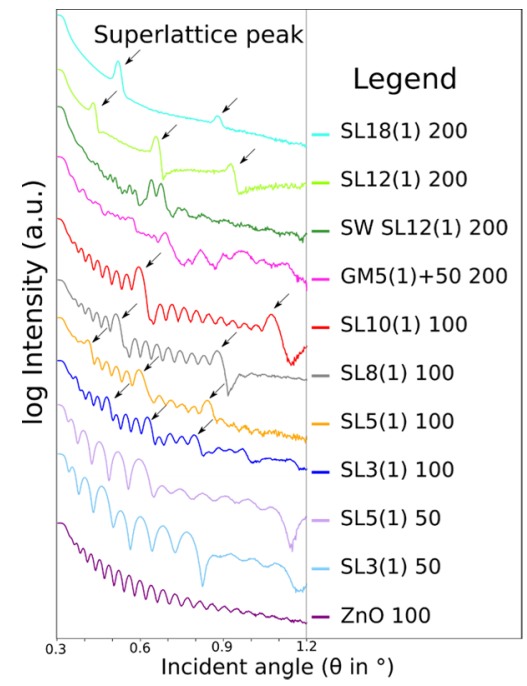

Figure 2. XRR patterns of selected samples; the arrows indicate SL peaks.

for the $200 \mathrm{~nm}$ thick samples even though the individual peaks become too small to be reliably distinguished from one another. The total film thickness of the films was obtained from these XRR patterns.

Figure 3 summarizes the thermal conductivities of both the new samples and those previously reported by us in the same
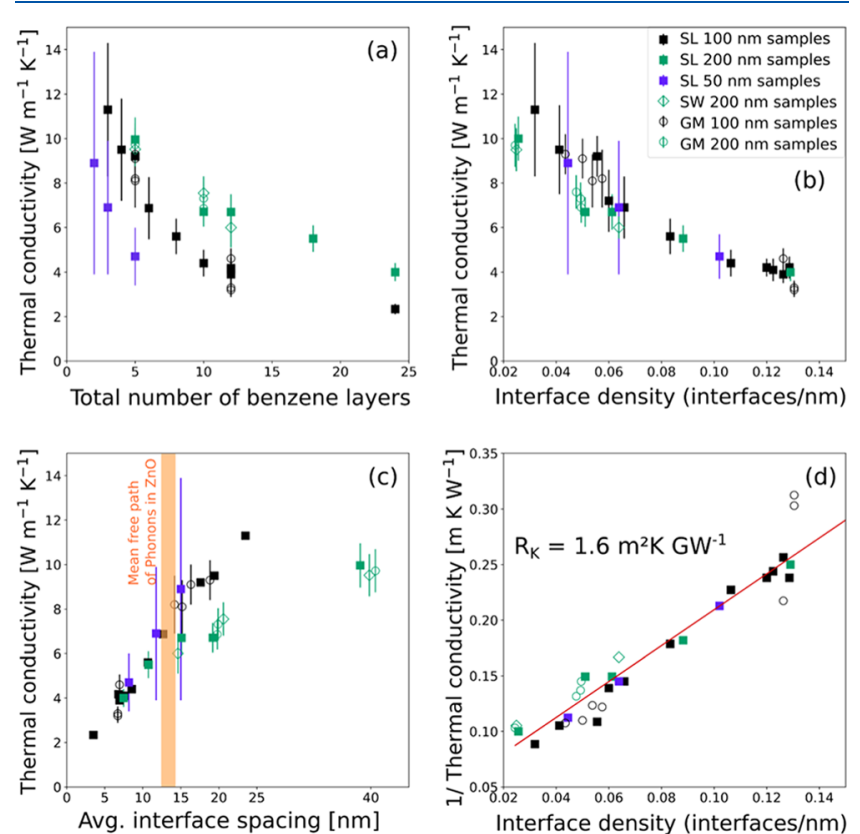

Figure 3. Thermal conductivity plotted against (a) total number of benzene layers, (b) interface density, and (c) average interface spacing. (d) Inverse thermal conductivity plotted against interface density; the plots include data from our previous publications. ${ }^{28,45,46}$

material system. ${ }^{28,45,46}$ Pure $\mathrm{ZnO}$ films deposited with ALD have a thermal conductivity above $40 \mathrm{Wm} / \mathrm{K}$ at $300 \mathrm{~K}^{64}$ Figure 3a shows that, adding the benzene layers within the $\mathrm{ZnO}$ matrix leads to a significant suppression of the thermal conductivity. This means the benzene layers act as barriers for phonon transport. The thermal conductivity systematically decreases with the total number of benzene layers. However, a clear split of the data is seen according to the overall film thickness, indicating that the absolute number of inorganicorganic interfaces is not the parameter that controls the suppression of thermal conductivity. A thicker film has a lower interface density and longer stretches for phonons to travel without interface scattering as a thinner film with the same number of interfaces. Therefore, in Figure $3 b$, we plot thermal conductivity against interface density, i.e., number of interfaces per nm film thickness instead of the total number of interfaces. The fact that the full dataset in Figure $3 \mathrm{~b}$ essentially forms a single curve that follows a monotonic decrease in thermal conductivity with increasing interface density is a strong indication that the interface density is the major factor controlling the reduction of thermal conductivity in our $\mathrm{ZnO}-$ benzene system. For the SL samples, this naturally translates into the conclusion that thermal conductivity is inversely dependent on the period length of the superlattices and incoherent phonon scattering dictates their thermal conductivity (compare, e.g., refs 7,65 ). This trend is in line with many inorganic-inorganic SL systems, on which, e.g., Norris et al. ${ }^{2}$ give an excellent overview.

In Figure 3c, we explore the relation between the thermal conductivity and the average spacing between the benzene layers (= average $\mathrm{ZnO}$-layer thickness); in this plot, we also mark the estimated mean free path of phonons of 13-14 nm calculated under the gray medium approximation (see the Supporting Information). Interestingly, around that average interface spacing of $13 \mathrm{~nm}$, a slight flattening of the curve seems to occur, which could indicate that at lower interface densities, other effects such as intrinsic phonon-phonon scattering in the $\mathrm{ZnO}$ layers start to play a role. For these larger average interface spacings $(>13 \mathrm{~nm}$ ), the $200 \mathrm{~nm}$ thick samples have slightly but consistently lower thermal conductivity than their $100 \mathrm{~nm}$ counterparts. Furthermore, the average spacing rather than individual differences in benzene-layer spacing has a more pronounced effect on the thermal conductivity of these SLs. Samples such as GM10(1)+18 $200 \mathrm{~nm}$ and SW SL12(1) $200 \mathrm{~nm}$ show the same thermal conductivity (within experimental uncertainties) as the superlattices SL12(1) 200 $\mathrm{nm}$ and SL10(1) $200 \mathrm{~nm}$, even though individual spacing between benzene layers is much smaller in the GM- and SWtype samples (see Figure 1). This also suggests that the overall film thickness could affect the thermal conductivity for samples with low interface densities.

In Figure 3d, we plot the inverse thermal conductivity against the interface density and calculate the Kapitza resistance to be $1.6 \pm 0.3 \mathrm{~m}^{2} \mathrm{~K} / \mathrm{GW}$; given that each benzene layer has a thickness of only one single benzene molecule, each layer is counted as one interface. This value is in line with a value reported already in our previous work ${ }^{28}$ with fewer data points and slightly higher than the value obtained, e.g., for inorganic crystalline/amorphous $\mathrm{ZnO} / \mathrm{InGaZnO}_{4}$ thin films $\left(1.35 \mathrm{~m}^{2} \mathrm{~K} / \mathrm{GW}\right){ }^{27}$ However, this is still below the theoretically possible Kapitza resistances across hybrid interfaces. $^{66}$

Part of the reason could be that the samples plotted in Figure 3 all have one thing in common, that is, the organic benzene layers were deposited with only one single MLD cycle. However, these monomolecular benzene layers may not completely separate the $\mathrm{ZnO}$ blocks, as they are likely to allow some $\mathrm{Zn}$ atoms to infiltrate within the organic layer. ${ }^{63} \mathrm{In}$ Figure 4, we thus compare samples that have thicker benzenelayer blocks (more than one consecutive MLD cycle, labeled "thick") with those with monomolecular benzene layers 


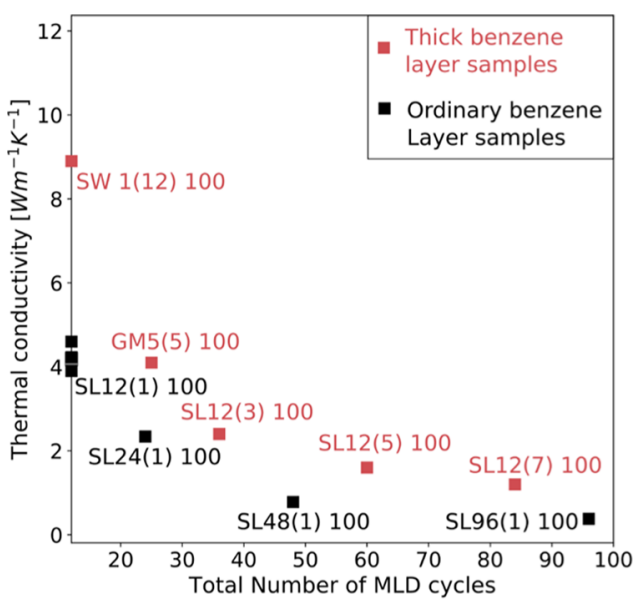

Figure 4. Thermal conductivity of thick samples with thicker benzene layers (in red) in comparison to ordinary samples with selected monomolecular-thick benzene layers (in black); the plot includes data from our previous publications. ${ }^{28,45,46}$

(labeled "ordinary"). This comparison is made so that we plot thermal conductivity against the total number of MLD cycles applied during the sample deposition; in the samples marked in black, each benzene layer is one MLD cycle thin, while in the thick samples, marked in red, more consecutive MLD cycles were applied for the individual benzene-layer blocks. From here, we can conclude that for the samples with the fixed number of benzene layers, the thicker the benzene layer, the lower the thermal conductivity; this is seen by comparing the four $100 \mathrm{~nm}$ thick samples, SL12(1), SL12(3), SL12(5), and SL12(7), for which the number of benzene layers is 12 and the individual benzene-layer thickness increases from one to seven MLD cycles (compare also ${ }^{28}$ ). Further comparison between the red and black symbols in Figure 4 shows that if we fix the overall number of MLD cycles to a certain number, lower thermal conductivity is achieved for the samples with 1 MLD cycle/benzene layer (black symbols) with more but thinner benzene layers. In other words, for a limited number of MLD cycles, the largest reduction in thermal conductivity can be achieved by using 1 MLD cycle/benzene layer increasing the interfacial density.

From the aforementioned discussion, it is apparent that many variables can have an influence on the thermal conductivity. To gain further understanding of the impacts of the different variables, we chose to employ a multivariate data analysis (MVDA) as a statistical tool to evaluate interdependencies between the variables and similarities between the samples. The selected PLS regression method reformulates our complex input dataset into a two-dimensional projection; this allows us to have an easy overview of the dataset, screen the variables, and-most importantly-show connections between the multiple variables, defined as $X$ - and $Y$-variables. ${ }^{62}$

Previously, we and others have successfully used this technique for magnetic, superconducting, and photovoltaic perovskite samples to address structure-property relations and also to enhance the characterization; in these cases, the datasets were larger, though. ${ }^{67-70}$ Here, our sample set is relatively small (39 samples) and the results must be taken with the respective care. However, the fact that the samples are similarly synthesized and analyzed increases the reliability of the analysis. We set thermal conductivity as the $Y$-variable, and for the $X$-variables, the following eight entries were selected
(Figure 5): total film thickness, benzene/ $\mathrm{ZnO}$ interface density, total number of benzene layers, benzene-layer

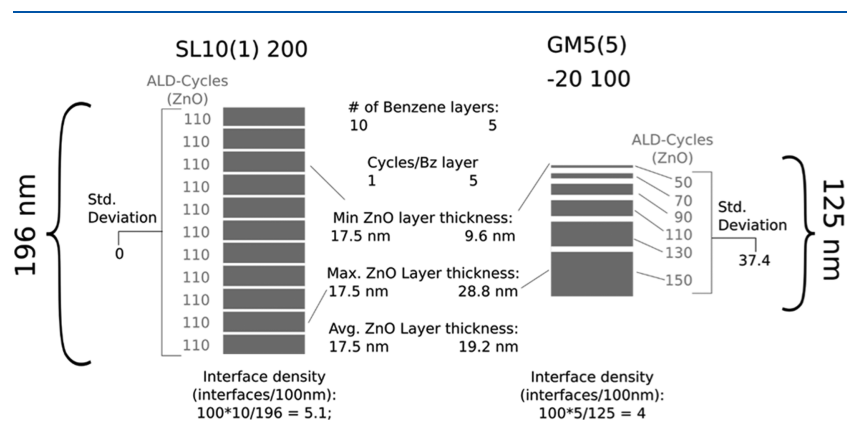

Figure 5. Visual explanations of the eight $X$-variables used for the multivariate analysis, shown via two example sketches.

thickness, average thickness of the $\mathrm{ZnO}$ layers, minimum $\mathrm{ZnO}$-layer thickness, maximum $\mathrm{ZnO}$-layer thickness, and the standard deviation of $\mathrm{ZnO}$ sublayer ALD cycles (as a quantifying measure for the variation range within the sample). The samples were divided into three classes based on the layer sequence type: samples with regular spacings ("SL"), samples with a gradient in the $\mathrm{ZnO}$ sublayers ("GM"), and samples with the organic layer thicker than one benzene layer (thick) to see whether there are any significant differences in behavior between samples of different layer pattern types.

Our PLS analysis converged on a two-axis representation of the original eight-variable system, making the sample presentation two-dimensional (Figure 6). In this plot, we can

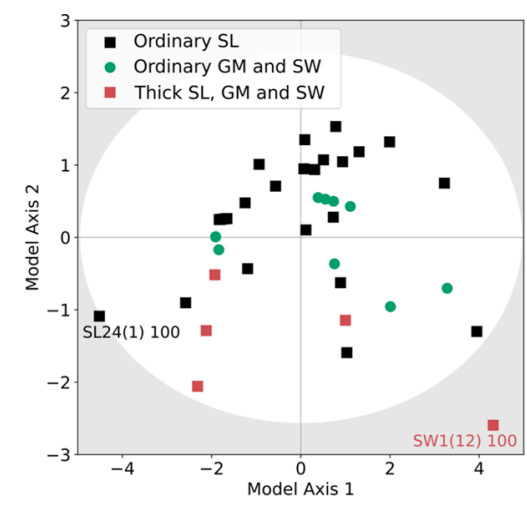

Figure 6. So-called scores plot showing the position of each sample on the two-dimensional projection plane. The ellipse represents socalled Hotelling's $T^{2}$ limit of $95 \%$ confidence.

see no discernible separation between the different sample groups, aside from a slight preference of the thick samples to place on the lower half of the plot. This overall homogeneous distribution means that there is no fundamental difference between the regular SL and the irregular GM structures. Furthermore, we note that the majority of the samples fall quite evenly within the ellipse of $95 \%$ confidence, and the two samples located outside of the ellipse are not too far from it. This confirms that the chosen sample set is well balanced and representative, and the chosen $X$-variables have managed to capture the variance among the samples well. A closer look on the two samples on the border or outside of the ellipse, namely, SL24(1) 100 and SW1(12) 100, reveals that those are the samples with the highest (SL24(1) 100) and lowest (SW1(12) 100) interface density, respectively, and SW1(12) 
100 also has the highest number of benzene cycles in only one layer.

The coefficients obtained for each original variable upon the data projection are illustrated in Figure 7. A large positive value

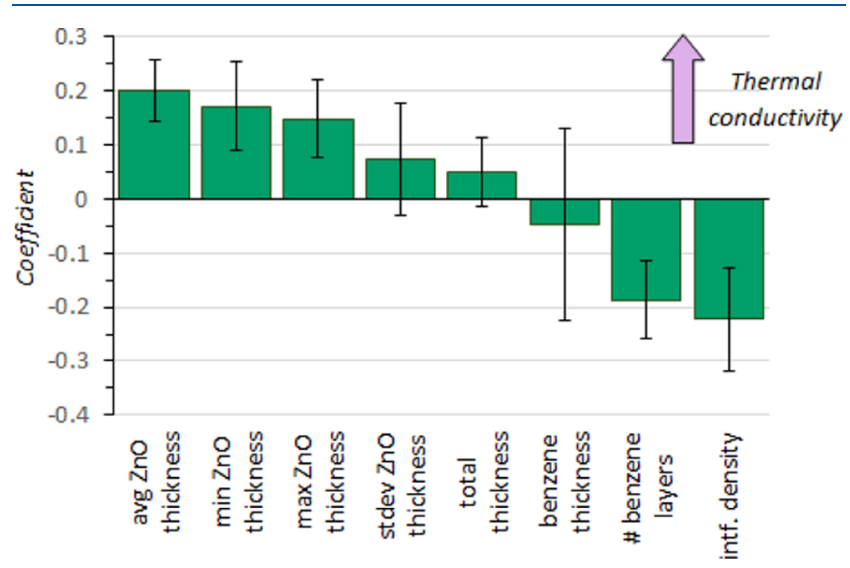

Figure 7. Coefficients received by each variable in the PLS regression analysis, represented as the height of the column; the line segment indicates the uncertainty. The arrow shows the direction of positive correlation with thermal conductivity.

means that increasing this specific variable will significantly increase the thermal conductivity, while a negative value implies the desired suppression of thermal conductivity. We note that the variables expressing average, maximum, and minimum ZnO-layer thickness have large positive values, meaning they correlate strongly with thermal conductivity, but to the incremental direction. Meanwhile, the interface-related variables (interface density and number of benzene layers) have highly negative values, indicating they have a strong inverse correlation with the thermal conductivity. All of these variables with large coefficients have relatively small uncertainty limits, reflecting the high reliability of the detected correlations. On the contrary, the variables for total sample thickness, standard deviation of the $\mathrm{ZnO}$-layer thickness, and benzene-layer thickness all express small weights and large error bars, which strongly suggest that these variables have less impact on thermal conductivity. Of these, the benzene-layer thickness has an especially large uncertainty, indicating that the detected correlation is possibly of random origin, presumably due to the small number of the thick samples.

Due to the relatively small number of samples and variables in general, the error bars easily become large. However, even when considering the uncertainty of the received coefficient values, we see a clear distinction into two groups based on the absolute values of the coefficients: those of the five most significant variables are consistently more than double those of the remaining three variables. Additionally, the three variables of lowest significance all present error bars surpassing their own total value. Shown in Figure 7 , the five variables thus determined to be highly meaningful are (in the order of importance) interface density, average $\mathrm{ZnO}$-layer thickness, total number of benzene layers, benzene-layer thickness, and the minimum and maximum $\mathrm{ZnO}$-layer thicknesses.

Finally, the relevance of our MVDA approach can be tested by evaluating if the analysis can predict the correct thermal conductivities based on the chosen eight $X$-variables. For finding the predicted thermal conductivity, each sample is defined by its position on the two-dimensional projection plane, and then the corresponding position on the $y$-axis is taken as the predicted thermal conductivity (akin to using a trendline to determine a property expressed on the $y$-axis). When plotting the predicted values against the measured values in Figure 8, we note that most samples follow the

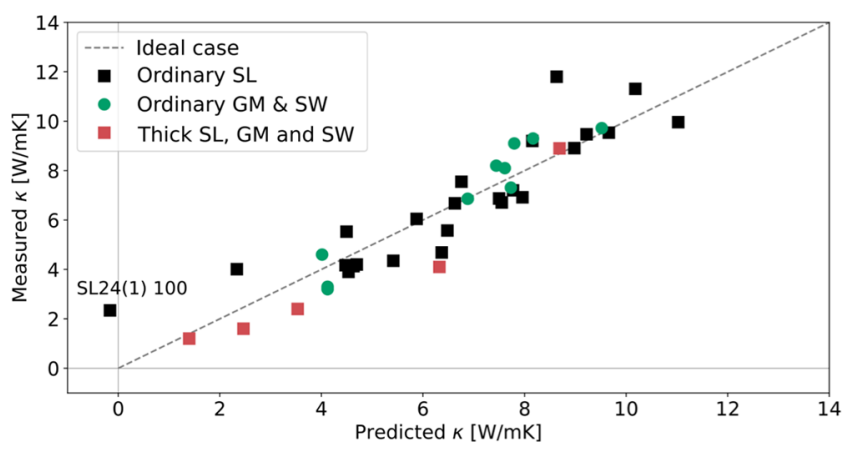

Figure 8. Experimental vs predicted thermal conductivity.

diagonal very well. This illustrates the good predictive power of the model, also evidenced by the commendable goodness-of-fit parameters $(\mathrm{R} 2 \mathrm{Y}=0.842 ; \mathrm{Q} 2=0.804)$. Only one deviating sample is singled out by a slightly negative predicted thermal conductivity value; a closer investigation points to the exceptionally high interface density of this SL24(1) 100 sample, setting it apart from the other samples.

\section{CONCLUSIONS}

We have used our ALD/MLD-fabricated $\mathrm{ZnO}$-benzene thin films as a model system to investigate the contributions of different factors affecting thermal conductivity in multilayered thin films. The sample set investigated constituted of 39 thinfilm samples with an overall thickness of 50-200 nm, and the thermal conductivity was determined using cross-plane TDTR measurements. A typical sample constituted of monomolecular benzene layers acting as phonon barrier in a polycrystalline $\mathrm{ZnO}$ matrix, but we also investigated the effect of increasing the thickness of these organic benzene layers. The remarkable advantage of the ALD/MLD technique is that it allows us to fabricate the $\mathrm{ZnO}-$ benzene films according to any predesigned benzene-layer dispersion pattern and thereby precisely control the individual $\mathrm{ZnO}$-layer thicknesses. Both regular and irregular dispersion patterns were investigated.

The fact that the sample fabrication and characterization scheme was essentially identical thorough the sample set investigated guaranteed the reliability of the data. This together with the appreciably large number of samples investigated allowed us to utilize a statistical multivariate data analysis approach to evaluate the correlations among the data and investigate the factors controlling the thermal conductivity. Altogether, eight structural/dimensional parameters describing the multilayer structures of the films were included in the analysis. It was verified that the interface density is the dominating factor. At very low interface densities, the decrease in thermal conductivity with increasing interface density was less pronounced; moreover, in this range, the overall film thickness seemed to somewhat differentiate the samples. The stronger reduction of the thermal conductivity observed in thicker benzene layers was attributed to a better separation between the $\mathrm{ZnO}$ blocks.

We believe that the understanding gained in this work on the control of thermal conductivity in our $\mathrm{ZnO}-$ benzene 
system is transferable to other multilayer thin-film systems. Moreover, we hope that our work motivates the use of multivariate analysis techniques in investigating various structure-property correlations in materials science in general.

\section{ASSOCIATED CONTENT}

\section{(3) Supporting Information}

The Supporting Information is available free of charge at https://pubs.acs.org/doi/10.1021/acs.jpcc.0c06461.

ALD/MLD cycle sequences used for sample deposition (Table S1); a brief estimation of the phonon mean free path in $\mathrm{ZnO}$; all known numerical values for the thermal conductivities plotted in Figure 3 (Table S2); and information on the choice of TDTR frequency and resulting sensitivity toward the sample (PDF)

\section{AUTHOR INFORMATION}

\section{Corresponding Author}

Maarit Karppinen - Department of Chemistry and Materials

Science, Aalto University, Fi-00076 Aalto, Finland;

(1) orcid.org/0000-0003-1091-1169;

Email: maarit.karppinen@aalto.fi

\section{Authors}

Fabian Krahl - Department of Chemistry and Materials Science, Aalto University, Fi-00076 Aalto, Finland

Ashutosh Giri - Department of Mechanical and Aerospace Engineering, University of Virginia, Charlottesville, Virginia 22904, United States; Department of Mechanical, Industrial and Systems Engineering, University of Rhode Island, Kingston, Rhode Island 02881, United States; orcid.org/0000-00028899-4964

Md Shafkat Bin Hoque - Department of Mechanical and Aerospace Engineering, University of Virginia, Charlottesville, Virginia 22904, United States

Linda Sederholm - Department of Chemistry and Materials Science, Aalto University, Fi-00076 Aalto, Finland

Patrick E. Hopkins - Department of Mechanical and Aerospace Engineering, Department of Materials Science and Engineering, and Department of Physics, University of Virginia, Charlottesville, Virginia 22904, United States; 이이.org/ 0000-0002-3403-743X

Complete contact information is available at: https://pubs.acs.org/10.1021/acs.jpcc.0c06461

\section{Notes}

The authors declare no competing financial interest.

\section{ACKNOWLEDGMENTS}

The authors acknowledge the funding from European Research Council under the European Union's Seventh Framework Programme (FP/2007-2013)/ERC Advanced Grant Agreement (339478) and Academy of Finland (296299) and the use of the RawMatTERS Finland Infrastructure (RAMI) and OtaNano-Nanomicroscopy Center (Aalto-NMC) at Aalto University. Sakari Lepikko is thanked for help in ellipsometry measurements. The authors appreciate support from the Army Research Office, Grant No. W911NF-16-1-0406.

\section{REFERENCES}

(1) Snyder, G. J.; Toberer, E. S. Complex Thermoelectric Materials. Nat. Mater. 2008, 7, 105-114.
(2) Norris, P. M.; Le, N. Q.; Baker, C. H. Tuning Phonon Transport: From Interfaces to Nanostructures. J. Heat Transfer 2013, 135, No. 061604.

(3) Kittel, C. Introduction to Solid State Physics, 8th ed.; John Wiley \& Sons, Inc: New York, 2005.

(4) Kelly, M. J. Acoustic Phonon Transmission in Superlattices. J. Phys. C: Solid State Phys. 1985, 18, 5963.

(5) Narayanamurti, V.; Störmer, H. L.; Chin, M. A.; Gossard, A. C.; Wiegmann, W. Selective Transmission of High-Frequency Phonons by a Superlattice: The "Dielectric" Phonon Filter. Phys. Rev. Lett. 1979, 43, 2012-2016.

(6) Kothari, K.; Maldovan, M. Phonon Surface Scattering and Thermal Energy Distribution in Superlattices. Sci. Rep. 2017, 7, No. 5625.

(7) Ravichandran, J.; Yadav, A. K.; Cheaito, R.; Rossen, P. B.; Soukiassian, A.; Suresha, S. J.; Duda, J. C.; Foley, B. M.; Lee, C.-H.; Zhu, Y.; et al. Crossover from Incoherent to Coherent Phonon Scattering in Epitaxial Oxide Superlattices. Nat. Mater. 2014, 13, $168-172$.

(8) Luckyanova, M. N.; Garg, J.; Esfarjani, K.; Jandl, A.; Bulsara, M. T.; Schmidt, A. J.; Minnich, A. J.; Chen, S.; Dresselhaus, M. S.; Ren, Z.; et al. Coherent Phonon Heat Conduction in Superlattices. Science 2012, 338, 936-939.

(9) Costescu, R. M.; Cahill, D. G.; Fabreguette, F. H.; Sechrist, Z. A.; George, S. M. Ultra-Low Thermal Conductivity in $\mathrm{W} / \mathrm{Al}_{2} \mathrm{O}_{3}$ Nanolaminates. Science 2004, 303, 989-990.

(10) Cahill, D. G.; Bullen, A.; Lee, S.-M. Interface Thermal Conductance and the Thermal Conductivity of Multilayer Thin Films. High Temp. - High Pressures 2000, 32, 135-142.

(11) Song, D. W.; Liu, W. L.; Zeng, T.; Borca-Tasciuc, T.; Chen, G.; Caylor, J. C.; Sands, T. D. Thermal Conductivity of Skutterudite Thin Films and Superlattices. Appl. Phys. Lett. 2000, 77, 3854-3856.

(12) Koh, Y. K.; Cao, Y.; Cahill, D. G.; Jena, D. Heat-Transport Mechanisms in Superlattices. Adv. Funct. Mater. 2009, 19, 610-615.

(13) Hołuj, P.; Euler, C.; Balke, B.; Kolb, U.; Fiedler, G.; Müller, M. M.; Jaeger, T.; Chávez Angel, E.; Kratzer, P.; Jakob, G. Reduced Thermal Conductivity of TiNiSn/HfNiSn Superlattices. Phys. Rev. B 2015, 92, No. 125436.

(14) Koga, T.; Cronin, S. B.; Dresselhaus, M. S.; Liu, J. L.; Wang, K. L. Experimental Proof-of-Principle Investigation of Enhanced Z3DT in (001) Oriented Si/Ge Superlattices. Appl. Phys. Lett. 2000, 77, $1490-1492$.

(15) Huxtable, S. T.; Abramson, A. R.; Tien, C.-L.; Majumdar, A.; LaBounty, C.; Fan, X.; Zeng, G.; Bowers, J. E.; Shakouri, A.; Croke, E. T. Thermal Conductivity of $\mathrm{Si} / \mathrm{SiGe}$ and $\mathrm{SiGe} / \mathrm{SiGe}$ Superlattices. Appl. Phys. Lett. 2002, 80, 1737-1739.

(16) Borca-Tasciuc, T.; Liu, W.; Liu, J.; Zeng, T.; Song, D. W.; Moore, C. D.; Chen, G.; Wang, K. L.; Goorsky, M. S.; Radetic, T.; et al. Thermal Conductivity of Symmetrically Strained Si/Ge Superlattices. Superlattices Microstruct. 2000, 28, 199-206.

(17) Lee, S.-M.; Cahill, D. G.; Venkatasubramanian, R. Thermal Conductivity of Si-Ge Superlattices. Appl. Phys. Lett. 1997, 70, 29572959.

(18) Chakraborty, S.; Kleint, C. A.; Heinrich, A.; Schneider, C. M.; Schumann, J.; Falke, M.; Teichert, S. Thermal Conductivity in Strain Symmetrized Si/Ge Superlattices on $\mathrm{Si}(111)$. Appl. Phys. Lett. 2003, 83, 4184-4186.

(19) Capinski, W. S.; Maris, H. J.; Ruf, T.; Cardona, M.; Ploog, K.; Katzer, D. S. Thermal-Conductivity Measurements of GaAs/AlAs Superlattices Using a Picosecond Optical Pump-and-Probe Technique. Phys. Rev. B 1999, 59, 8105-8113.

(20) Luckyanova, M. N.; Johnson, J. A.; Maznev, A. A.; Garg, J.; Jandl, A.; Bulsara, M. T.; Fitzgerald, E. A.; Nelson, K. A.; Chen, G. Anisotropy of the Thermal Conductivity in GaAs/AlAs Superlattices. Nano Lett. 2013, 13, 3973-3977.

(21) Venkatasubramanian, R. Lattice Thermal Conductivity Reduction and Phonon Localizationlike Behavior in Superlattice Structures. Phys. Rev. B 2000, 61, 3091-3097. 
(22) Borca-Tasciuc, T.; Achimov, D.; Liu, W. L.; Chen, G.; Ren, H.W.; Lin, C.-H.; Pei, S. S. Thermal Conductivity of InAs/ AlSb Superlattices. Microscale Thermophys. Eng. 2001, 5, 225-231.

(23) Zhang, Y.; Chen, Y.; Gong, C.; Yang, J.; Qian, R.; Wang, Y. Optimization of Superlattice Thermoelectric Materials and Microcoolers. J. Microelectromech. Syst. 2007, 16, 1113-1119.

(24) Tong, H.; Miao, X. S.; Cheng, X. M.; Wang, H.; Zhang, L.; Sun, J. J.; Tong, F.; Wang, J. H. Thermal Conductivity of Chalcogenide Material with Superlatticelike Structure. Appl. Phys. Lett. 2011, 98, No. 101904

(25) Ali, S.; Juntunen, T.; Sintonen, S.; Ylivaara, O. M. E.; Puurunen, R. L.; Lipsanen, H.; Tittonen, I.; Hannula, S.-P. Thermal Conductivity of Amorphous $\mathrm{Al}_{2} \mathrm{O}_{3} / \mathrm{TiO}_{2}$ Nanolaminates Deposited by Atomic Layer Deposition. Nanotechnology 2016, 27, No. 445704.

(26) Giri, A.; Donovan, B. F.; Hopkins, P. E. Localization of Vibrational Modes Leads to Reduced Thermal Conductivity of Amorphous Heterostructures. Phys. Rev. Mater. 2018, 2, No. 056002.

(27) Krahl, F.; Wu, Y.; Cho, H. J.; Karppinen, M.; Ohta, H. Spontaneous Generation of Carrier Electrons at the Interface between Polycrystalline $\mathrm{ZnO}$ and Amorphous $\mathrm{InGaZnO}_{4}$. Adv. Electron. Mater. 2020, 6, No. 2000404.

(28) Giri, A.; Niemelä, J.-P.; Tynell, T.; Gaskins, J. T.; Donovan, B. F.; Karppinen, M.; Hopkins, P. E. Heat-Transport Mechanisms in Molecular Building Blocks of Inorganic/Organic Hybrid Superlattices. Phys. Rev. B 2016, 93, No. 115310.

(29) Karttunen, A. J.; Tynell, T.; Karppinen, M. Atomic-Level Structural and Electronic Properties of Hybrid Inorganic-Organic ZnO:Hydroquinone Superlattices Fabricated by ALD/MLD. J. Phys. Chem. C 2015, 119, 13105-13114.

(30) Karttunen, A. J.; Tynell, T.; Karppinen, M. Layer-by-Layer Design of Nanostructured Thermoelectrics: First-Principles Study of ZnO:Organic Superlattices Fabricated by ALD/MLD. Nano Energy 2016, 22, 338-348.

(31) Tian, R.; Wan, C.; Wang, Y.; Wei, Q.; Ishida, T.; Yamamoto, A.; Tsuruta, A.; Shin, W.; Li, S.; Koumoto, K. A Solution-Processed $\mathrm{TiS}_{2}$ /Organic Hybrid Superlattice Film towards Flexible Thermoelectric Devices. J. Mater. Chem. A 2017, 5, 564-570.

(32) Wan, C.; Gu, X.; Dang, F.; Itoh, T.; Wang, Y.; Sasaki, H.; Kondo, M.; Koga, K.; Yabuki, K.; Snyder, G. J.; et al. Flexible N-Type Thermoelectric Materials by Organic Intercalation of Layered Transition Metal Dichalcogenide $\mathrm{TiS}_{2}$. Nature Mater. 2015, 14, 622-627.

(33) Wan, C.; Tian, R.; Kondou, M.; Yang, R.; Zong, P.; Koumoto, K. Ultrahigh Thermoelectric Power Factor in Flexible Hybrid Inorganic-Organic Superlattice. Nat. Commun. 2017, 8, No. 1024.

(34) Wan, C.; Tian, R.; Azizi, A. B.; Huang, Y.; Wei, Q.; Sasai, R.; Wasusate, S.; Ishida, T.; Koumoto, K. Flexible Thermoelectric Foil for Wearable Energy Harvesting. Nano Energy 2016, 30, 840-845.

(35) Sundberg, P.; Karppinen, M. Organic and Inorganic-Organic Thin Film Structures by Molecular Layer Deposition: A Review. Beilstein J. Nanotechnol. 2014, 5, 1104-1136.

(36) Nilsen, O.; Klepper, K.; Nielsen, H.; Fjellvåg, H. Deposition of Organic- Inorganic Hybrid Materials by Atomic Layer Deposition. ECS Trans. 2019, 16, 3-14.

(37) Suntola, T. Atomic Layer Epitaxy. Mater. Sci. Rep. 1989, 4, $261-312$.

(38) Ohtaki, M.; Tsubota, T.; Eguchi, K.; Arai, H. High-temperature Thermoelectric Properties of $\left(\mathrm{Zn}_{1-x} \mathrm{Al}_{\mathrm{x}}\right)$ O. J. Appl. Phys. 1996, 79, $1816-1818$.

(39) Tsubota, T.; Ohtaki, M.; Eguchi, K.; Arai, H. Transport Properties and Thermoelectric Performance of $\left(\mathrm{Zn}_{1-y} \mathrm{Mg}_{\mathrm{y}}\right)_{1-\mathrm{x}} \mathrm{Al}_{\mathrm{x}} \mathrm{O}$. J. Mater. Chem. 1998, 8, 409-412.

(40) Ohtaki, M.; Araki, K.; Yamamoto, K. High Thermoelectric Performance of Dually Doped ZnO Ceramics. J. Electron. Mater. 2009, 38, 1234-1238.

(41) Katsuyama, S.; Takagi, Y.; Ito, M.; Majima, K.; Nagai, H.; Sakai, H.; Yoshimura, K.; Kosuge, K. Thermoelectric Properties of $\left(\mathrm{Zn}_{1-\mathrm{y}} \mathrm{Mg}_{\mathrm{y}}\right)_{1-\mathrm{x}} \mathrm{Al}_{\mathrm{x}} \mathrm{O}$ Ceramics Prepared by the Polymerized Complex Method. J. Appl. Phys. 2002, 92, 1391-1398.
(42) Cai, K. F.; Müller, E.; Drašar, C.; Mrotzek, A. Preparation and Thermoelectric Properties of Al-Doped ZnO Ceramics. Mater. Sci. Eng. B 2003, 104, 45-48.

(43) Tynell, T.; Karppinen, M. Atomic Layer Deposition of ZnO: A Review. Semicond. Sci. Technol. 2014, 29, No. 043001.

(44) Özgür, Ü.; Alivov, Y.; Liu, C.; Teke, A.; Reshchikov, M. A.; Doğan, S.; Avrutin, V.; Cho, S.-J.; Morkoç, H. A Comprehensive Review of $\mathrm{ZnO}$ Materials and Devices. J. Appl. Phys. 2005, 98, No. 041301.

(45) Tynell, T.; Giri, A.; Gaskins, J.; Hopkins, P. E.; Mele, P.; Miyazaki, K.; Karppinen, M. Efficiently Suppressed Thermal Conductivity in $\mathrm{ZnO}$ Thin Films via Periodic Introduction of Organic Layers. J. Mater. Chem. A 2014, 2, 12150-12152.

(46) Krahl, F.; Giri, A.; Tomko, J. A.; Tynell, T.; Hopkins, P. E.; Karppinen, M. Thermal Conductivity Reduction at InorganicOrganic Interfaces: From Regular Superlattices to Irregular Gradient Layer Sequences. Adv. Mater. Interfaces 2018, 5, No. 1701692.

(47) Liu, J.; Yoon, B.; Kuhlmann, E.; Tian, M.; Zhu, J.; George, S. M.; Lee, Y.-C.; Yang, R. Ultralow Thermal Conductivity of Atomic/ Molecular Layer-Deposited Hybrid Organic-Inorganic Zincone Thin Films. Nano Lett. 2013, 13, 5594-5599.

(48) Yoon, B.; Lee, B. H.; George, S. M. Highly Conductive and Transparent Hybrid Organic-Inorganic Zincone Thin Films Using Atomic and Molecular Layer Deposition. J. Phys. Chem. C 2012, 116, 24784-24791.

(49) Karttunen, A. J.; Sarnes, L.; Townsend, R.; Mikkonen, J.; Karppinen, M. Flexible Thermoelectric $\mathrm{ZnO}-$ Organic Superlattices on Cotton Textile Substrates by ALD/MLD. Adv. Electron. Mater. 2017, 3, No. 1600459.

(50) Marin, G.; Tynell, T.; Karppinen, M. Flexible Thermoelectric Modules Based on ALD-Grown ZnO on Different Substrates. J. Vac. Sci. Technol., A 2019, 37, No. 020906.

(51) Marin, G.; Funahashi, R.; Karppinen, M. Textile-Integrated ZnO-Based Thermoelectric Device Using Atomic Layer Deposition. Adv. Eng. Mater. 2020, No. 2000535.

(52) Niemelä, J.-P.; Giri, A.; Hopkins, P. E.; Karppinen, M. UltraLow Thermal Conductivity in $\mathrm{TiO}_{2}: \mathrm{C}$ Superlattices. J. Mater. Chem. A 2015, 3, 11527-11532.

(53) Niemelä, J.-P.; Karttunen, A. J.; Karppinen, M. InorganicOrganic Superlattice Thin Films for Thermoelectrics. J. Mater. Chem. C 2015, 3, 10349-10361.

(54) Ghiyasi, R.; Tewari, G. C.; Karppinen, M. Organic-Component Dependent Crystal Orientation and Electrical Transport Properties in ALD/MLD Grown ZnO-Organic Superlattices. J. Phys. Chem. C 2020, 124, 13765-13770.

(55) Cahill, D. G. Analysis of Heat Flow in Layered Structures for Time-Domain Thermoreflectance. Rev. Sci. Instrum. 2004, 75, 51195122.

(56) Jiang, P.; Qian, X.; Yang, R. Tutorial: Time-Domain Thermoreflectance (TDTR) for Thermal Property Characterization of Bulk and Thin Film Materials. J. Appl. Phys. 2018, 124, No. 161103.

(57) Schmidt, A. J.; Chen, X.; Chen, G. Pulse Accumulation, Radial Heat Conduction, and Anisotropic Thermal Conductivity in PumpProbe Transient Thermoreflectance. Rev. Sci. Instrum. 2008, 79, No. 114902.

(58) Giri, A.; Niemelä, J.-P.; Szwejkowski, C. J.; Karppinen, M.; Hopkins, P. E. Reduction in Thermal Conductivity and Tunable Heat Capacity of Inorganic/Organic Hybrid Superlattices. Phys. Rev. B 2016, 93, No. 024201.

(59) Wold, S.; Ruhe, A.; Wold, H.; Dunn, I. W. J. The Collinearity Problem in Linear Regression. The Partial Least Squares (PLS) Approach to Generalized Inverses. SIAM J. Sci. Stat. Comput. 1984, 5, $735-743$.

(60) Kettaneh-Wold, N. Analysis of Mixture Data with Partial Least Squares. Chemom. Intell. Lab. Syst. 1992, 14, 57-69.

(61) Rosipal, R.; Krämer, N. Overview and Recent Advances in Partial Least Squares. In Subspace, Latent Structure and Feature Selection;Saunders, C.; Grobelnik, M.; Gunn, S.; Shawe-Taylor, J., 
Eds.; Springer: Berlin, Heidelberg, 2006; pp 34-51. https://doi.org/ 10.1007/11752790_2.

(62) Eriksson, L.; Byrne, T.; Johansson, E.; Trygg, J.; Vikström, C. Multi- and Megavariate Data Analysis Basic Principles and Applications; Umetrics Academy: Malmö, 2013.

(63) Niemelä, J.-P.; Aghaee, M.; Kessels, W. M. M. E.; Creatore, M.; Verheijen, M. A. Transition in Layer Structure of Atomic/Molecular Layer Deposited ZnO-Zincone Multilayers. J. Vac. Sci. Technol., A 2019, 37, No. 040602.

(64) Alvarez-Quintana, J.; Martínez, E.; Pérez-Tijerina, E.; PérezGarcía, S. A.; Rodríguez-Viejo, J. Temperature Dependent Thermal Conductivity of Polycrystalline ZnO Films. J. Appl. Phys. 2010, 107, No. 063713.

(65) Cheaito, R.; Polanco, C. A.; Addamane, S.; Zhang, J.; Ghosh, A. W.; Balakrishnan, G.; Hopkins, P. E. Interplay between Total Thickness and Period Thickness in the Phonon Thermal Conductivity of Superlattices from the Nanoscale to the Microscale: Coherent versus Incoherent Phonon Transport. Phys. Rev. B 2018, 97, No. 085306.

(66) Giri, A.; Hopkins, P. E. A Review of Experimental and Computational Advances in Thermal Boundary Conductance and Nanoscale Thermal Transport across Solid Interfaces. Adv. Funct. Mater. 2020, 30, No. 1903857.

(67) Lehmus, K.; Karppinen, M. Application of Multivariate Data Analysis Techniques in Modeling Structure-Property Relationships of Some Superconductive Cuprates. J. Solid State Chem. 2001, 162, $1-9$.

(68) Imamura, N.; Mizoguchi, T.; Yamauchi, H.; Karppinen, M. Multivariate Data Analysis Approach to Understand Magnetic Properties of Perovskite Manganese Oxides. J. Solid State Chem. 2008, 181, 1195-1203.

(69) Tiittanen, T.; Vasala, S.; Karppinen, M. Assessment of Magnetic Properties of $\mathrm{A}_{2} \mathrm{~B}^{\prime} \mathrm{B}^{\prime \prime} \mathrm{O}_{6}$ Double Perovskites by Multivariate Data Analysis Techniques. Chem. Commun. 2019, 55, 1722-1725.

(70) Cacovich, S.; Matteocci, F.; Abdi-Jalebi, M.; Stranks, S. D.; Di Carlo, A.; Ducati, C.; Divitini, G. Unveiling the Chemical Composition of Halide Perovskite Films Using Multivariate Statistical Analyses. ACS Appl. Energy Mater. 2018, 1, 7174-7181. 